\title{
Behavioral Health Crisis Stabilization Centers: A New Normal
}

\author{
Verletta Saxon ${ }^{*}$, Dhrubodhi Mukherjee ${ }^{2}$, Deborah Thomas ${ }^{3}$ \\ 'Department of Children and Family Services, State of Illinois, Chicago, IL, United States \\ ${ }^{2}$ Associate Professor, Department of Social Work, University of North Texas, Denton, TX, United States
}

${ }^{3}$ Crisis Manager, Centerstone of Illinois, Carterville, IL, United States

Article Info

\section{Article Notes}

Received: March 15, 2018

Accepted: June 08, 2018

\section{${ }^{*}$ Correspondence:}

Dr. Verletta A. Saxon, Department of Children and Family Services, State of Illinois, Chicago, IL, United States;

E-mail: verlettas@gmail.com

(C) 2018 Saxon V. This article is distributed under the terms of the Creative Commons Attribution 4.0 International License.

\section{Keywords}

Crisis

Behavioral health

Psychological boarding

Psychiatric hospitals

Community behavioral health

Crisis stabilization centers

Living room model

Multidisciplinary teams

Emergency department

\section{ABSTRACT}

Shifting resources and funding from institutionalized care for those with mental illness to community-based care has shown promise for behavioral health parity in health crisis circumstances and yet, it has been underfunded. One of the unfortunate trends of deinstitutionalization of behavioral health services in general has been a persistent gap in emergency crisis services. This gap in services leaves those in a behavioral health crisis to receive treatment in the Hospital Emergency Departments culminating in an astounding increase in overall healthcare expenditures. Providing behavioral health crisis assessment and treatment in busy emergency departments that produce long waits for care can be a challenging environment for those in need of immediate treatment for psychological needs. Crisis Stabilization Centers are effective at providing suicide prevention services, addressing behavioral health treatment, diverting individuals from entering a higher level of care and addressing the distress experienced by individuals in a behavioral health crisis. Studies also show that the cost of Crisis Stabilization Centers is significantly less than psychiatric inpatient units and satisfaction among clients is greater. Expanding the options for Behavioral Health Crisis Care from community-based behavioral health outpatient care and inpatient care to various community alternatives, benefits individuals in crisis as well as the community. This article provides an overview of community alternatives to psychiatric hospitalization, financial barriers to care and future research.

\section{Introduction}

The Community Mental Health Act (1963), signed by President John F. Kennedy was the first federal policy that shifted funding and services from institutionalized settings to community-based behavioral health services. In the 55 years since President Kennedy's initiative to deinstitutionalize Psychiatric Hospitals, we have seen a growth in community-based behavioral health services in the United States ${ }^{1}$ (Action Alliance, 2016). This growth has incentivized community behavioral health centers, channelized funding to individuals with mental illness through Medicaid while addressing client's rights and experiences with the deinstitutionalization of Psychiatric Hospitals and creation of community-based services. More improvement in Communitybased Care is needed in order to provide adequate services to individuals experiencing a behavioral health crisis ${ }^{2}$ (Angar-Jacomb \& Read, 2009). Historically, states have been unable to provide sufficient resources and alternatives to psychiatric hospitalization for individuals experiencing a behavioral health crisis.

According to the ${ }^{3}$ Agency for Healthcare Research and Quality 
(2017), 1\% of all adult Emergency Department (ED) visits involved suicidal ideation, which is a serious behavioral health concern. For those who experience a behavioral health crisis, upon arrival at a hospital emergency department (ED) there is usually a long wait in a busy environment with other individuals experiencing treatment for severe medical complications. The person experiencing a behavioral health crisis will eventually receive a behavioral health assessment, an expensive bill and a referral to a Psychiatric Hospital or community service ${ }^{4,5}$ (Saxon, 2015; Mukherjee \& Saxon, 2017). These systems of care environments trigger symptoms and stress for those experiencing a behavioral health crisis $^{1}$ (Action Alliance, 2016). In 2017, often the services received were not sufficient, comprehensive or intense enough to meet the needs of individuals who were at risk of entering or exiting psychiatric care.

In order to improve care and reduce the frequency, cost, and length of stay (LOS) of ED visits for those in a behavioral health crisis, communities are creating Crisis Stabilization Centers $^{4}$ (Saxon, 2015). The National Action Alliance for Suicide Prevention (2016) considers Crisis Stabilization Centers to be a core element of Crisis Care. The Substance Abuse and Mental Health Services Administration (2014) defines Crisis Stabilization Services as:

A direct service that assists with deescalating the severity of a person's level of distress and/or need for urgent care associated with a substance use or mental health disorder. Crisis stabilization Services are designed to prevent or ameliorate a behavioral health crisis and/or reduce acute symptoms of mental illness by providing continuous 24-hour observation and supervision for persons who do not require inpatient services. Short-term crisis residential stabilization services include a range of community-based resources that can meet the needs of an individual with an acute psychiatric crisis and provide a safe environment for care and recovery (page 9).

Crisis Stabilization Services include telephone services, walk-in services, mobile crisis, short-term residential treatment, 23-hour Crisis Stabilization Units, the Living Room Model, Crisis Stabilization Units and psychiatric hospitalization. Crisis Stabilization Services provide safety and security for individuals in a psychiatric crisis and they can range from stand-alone sub-acute community-based units with length of stays from 1-10 days to hospitalbased systems with recliner chairs and 24-hour length of stays $^{6}$ (James \& Gilliland, 2001). These programs provide a range of services as an alternative to long term hospital stays and often allow the client to remain in their community to receive treatment services. Services provided can include assessment, case management, counseling, referrals, and linkage ${ }^{2}$ (Agar-Jacomb \& Read, 2009).
This article summarizes community-based behavioral health Crisis Stabilization Services, multidisciplinary teams, and financial barriers to providing quality services.

\section{Crisis Stabilization Center Models}

\section{3-Hour Crisis Stabilization}

23-hour crisis stabilization units offer an alternative to emergency department and psychiatric hospitalization admission by providing 23-hour crisis respite and observation in the community ${ }^{7}$ (SAMHSA 2014). The setting of this model resembles a home environment and offers assessment, rapid stabilization, reduction in crisis symptoms and observation in a community-based setting $^{8}$ (Thin et al, 2015). The model seeks to provide a safe environment, relieve crisis symptoms immediately, provide observation, determine level of care and to deflect from unnecessary higher levels of care $^{7,8}$ (SAMHSA, 2014; Thin et al, 2015). Evaluating the impact of the 23-hour crisis stabilization units showed effectiveness in deflecting individuals from psychiatric hospitalization, reduction in health care cost and improved treatment ${ }^{9,10,8}$ (Gillig et al., 1989; Francis et al., 2000; Thinn et al., 2015).

\section{The Living Room Model}

The Living Room Model is a walk-in respite centers for individuals in crisis. These home-like environments offer a courteous and calming surrounding for immediate relief of crisis symptoms and to avert psychiatric hospitalization ${ }^{11}$ (Heyland, Emery, \& Shattell, 2013). The goal of treatment in the Living Room Model is to provide a safe and secure environment where multidisciplinary professionals and peers with similar experiences provide treatment services. The Living Room Model highlights peers working or collaborating directly with clients to assist with symptom relief $^{1}$ (Action Alliance, 2016). The Living Room Model is distinctly different from the 23-hour crisis stabilization units. The Living Room Model provides crisis resolution and treatment for those who need more than 24 hours to resolve the issues that brought them into crisis, are short term and provide intensive treatment.

\section{Crisis Stabilization Centers}

Crisis Stabilization Centers (also known as short-term crisis residential stabilization services, community-based behavioral health stabilization, crisis stabilization, and crisis stabilization facilities) are home-like environments that address behavioral health crisis in a communitybased behavioral health or hospital setting. They are bedded units that range from 6-16 beds and staffed by licensed and unlicensed peer support as well as clinical and non-clinical professionals who hold masters and bachelor degrees ${ }^{7,5}$ (SAMHSA, 2014; Mukherjee \& Saxon, 2017). Services may consist of assessment, 
diagnosis, abbreviated treatment planning, observation, case management, individual and group counseling, skills training, prescribing and monitoring of psychotropic medication, referral, and linkage. Service delivery is offered on a 24-hour basis to address the client's immediate safety needs, develop resilience and create a plan to address the cyclical nature of behavioral health challenges and future behavioral health crisis for adults and children. The National Alliance for Suicide Prevention (2016) considers Crisis Stabilization Centers to be a "core element" of behavioral health crisis systems. Different from the Living Room Model and the 23-Hour Crisis Stabilization Unit, Crisis Stabilization Centers offer services to individuals whose needs cannot be met in the community. The environment is safe and secure and less restrictive than a hospital setting.

In a recent study by Mukherjee and Saxon (2017), the authors reported on the creation of a model of care at a Crisis Stabilization Center in rural Illinois that implemented one of three models for deflecting individuals from increased levels of behavioral health care. In this model, clients entering the ED would receive a clinical assessment and on the basis of the assessment could be transferred to a community-based crisis center for treatment. The study showed the LOS in the ED decreased from 7.3 hours to 4.12 hours after the introduction of the behavioral health crisis stabilization center intervention. The study also conducted a cost-analysis that showed this intervention saved an approximate $\$ 4.1$ million in Medicaid cost.

In a separate study by Wilder Research (2013), a crisis stabilization unit in a metropolitan Minnesota area examined the impact of the unit on the ED, outpatient services and inpatient psychiatric service utilization. The study found the overall cost of providing services in a community-based crisis center was less than providing services in an inpatient unit.

\section{Multi-Disciplinary Team Approaches}

Mukherjee and Saxon (2017) found that one of the keys to developing Crisis Stabilization Centers is to work in multi-disciplinary teams. Crisis Intervention is provided by multiple entities which can include police, hospitals, nurses, ambulatory services, behavioral health and many other professionals ${ }^{6,5}$ (James \& Gilliland, 2001; Mukherjee \& Saxon, 2017). When we combine the cumulative knowledge, skills, and ability of partners that serve individuals in psychiatric crisis we achieve a panoply of interdisciplinary skillsets that address the needs of a comprehensive integrated behavioral healthcare system. When identifying key stakeholders the following agencies can be engaged: behavioral health, health care, substance abuse, children and family services, older adult services, ambulatory services, state, home health, employment services, women's centers, family planning,
Medicaid, Social Security, Health and Human Services, legal services, advocacy groups, education and federal leaders, public and private agencies ${ }^{11,1,5}$ (National Action Alliance for Suicide Prevention, 2011; National Action Alliance for Suicide Prevention, 2016; Mukherjee \& Saxon, 2017). Building a team that can collaborate and address the systematic and personal challenges of those experiencing a behavioral health crisis creates a more effective system that increases service delivery while reducing the overall health care cost for those in crisis $^{6}$ (James \& Gilliland, 2001). When collaboration among agencies and individuals is performed it reaches into political, local, state wide, federal, bureaucratic systems to create an environment where the voice of those who are suffering from psychiatric crisis can be ${ }^{6}$ heard (James \& Gilliland, 2001).

While multi-disciplinary teams improve the outcomes for individuals in crisis they also face challenges. Challenges to forming and sustaining multidisciplinary teams include selecting the most appropriate community providers to be a part of the team, the loss of funding that effect service delivery, agency turnover, inconsistent meeting dates and times, lack of communication, and the ability to provide adequate oversight of the client ${ }^{8,13}$ (Thinn, et al., 2015; Colombo, Bendelow, Fulford \& Williams, 2003). It is important to carefully consider the challenges to multidisciplinary teams as they are being formed and as the client progresses in treatment.

\section{Financial Barriers}

Crisis Stabilization Centers are a core part of the continuum of care for clients who experience a behavioral health crisis $^{7}$ (SAMHSA, 2014). When behavioral health crisis services exclusively rely on transitional funding such as grants, or they are tied to insurance requirements for care that narrowly define the prospective clients; this negatively impacts how crisis services are delivered, particularly in rural communities with smaller populations ${ }^{7}$ (SAMHSA, 2014). Private insurance companies have inflexible requirements for crisis services, resulting in states utilizing funding for indigent clients instead of funding through insurance. These pose challenging barriers to sustainable behavioral health crisis stabilization models of any sort. To be successful at providing individuals in crisis with supplemental services that will enhance treatment, there is a need to blend multiple categorical and singleservice funding sources and resources to address the diverse needs of this population ${ }^{14}$ (Collins et al., 2010).

\section{Future Research}

Heyland \& Johnson (2017) ${ }^{15}$ report that the need for variation in community-based treatment options for those in a behavioral health crisis still exist. The need for more treatment options coupled with funding cuts increases the number of individuals in crisis. This reduction in resources 
leaves emergency departments as the primary resource for psychiatric crisis services. Community alternatives for crisis care are a viable option for individuals in crisis. After reviewing 27 studies on the effectiveness of Crisis Stabilization Centers, the Action Alliance (2014) found that Crisis Stabilization Centers provide cost savings and are effective at treating individuals experiencing a behavioral health crisis. The authors encourage more research in this area including the connecting of funding opportunities that would address multiple streams of combined funding. Although preliminary outcomes on Crisis Stabilization Centers are positive, more research is needed to create outcomes and understand environmental challenges, continuous quality improvements, models of service, evidence-based approaches, outreach programs and multicultural issues.

\section{Conclusions}

Crisis Stabilization Centers are a viable alternative to Emergency Department behavioral health treatment. Research has shown that models such as 23-hour stabilization, the Living Room Model and Crisis Stabilization Centers have been shown to be effective at treating individuals in crisis and are cost effective. While communities create effective partnerships with federal, state and local administrative bodies they lack resources and funding to provide consistent treatment and improve on service delivery. At a time when the need for behavioral health service is in the national spotlight, behavioral health agencies and hospital systems are seeing the importance of new community-based crisis service delivery models and are addressing individuals in crisis.

\section{Conflicts of Interest}

The authors listed below declare that they do not have a conflict of interest:

\section{Verletta A. Saxon}

\section{Dhrubodhi Mukherjee}

The author listed below reports the following details of affiliation in an organization listed in this article:

Deborah Thomas is an employee of Centerstone of Illinois.

\section{References}

1. National Action Alliance for Suicide Prevention: Crisis Services Task Force. Crisis Now: Transforming Services is Within Our Reach. Washington, DC: Education Development Center, Inc. 2016.

2. Agar-Jacomb K, Read J. Mental health crisis services: What do service users need when in crisis? Journal of Mental Health. 2009); 18(2): 99110.
3. Agency for Healthcare Research and Quality. Emergency department visits related to suicidal ideation. 2017; 2006-2013. Retrieved from https://www.hcup-us.ahrq.gov/reports/statbriefs/sb220Suicidal-Ideation-ED-Visits.pdf. Accessed March4, 2018

4. Saxon V. Psychiatric Boarding: Evolving models for Community Crisis. 2015.

5. Mukherjee, D. \& Saxon, V. "Psychiatric Boarding" and challenges to community-based behavioral health crisis stabilization. Community Mental Health Journal Retrieved. 2018. from: https:// link.springer.com/epdf/10.1007/s10597-018-0237-9?author_access_token=b0oASxFNOpBmCZEpn8Y5QPe4RwlQNchNByi7wbcMAY4y5AaIvaFbzbItoLET5amUgsp86rColZwr_NSDp0bXmeWkmMiMJ4FNSNDvis0xTM0xia9Qq9R5946XIY_MplIvdaKzYyogMLtE1ITJu2uz1w\%3D\%3D

6. James RK, Gilliland BE. Crisis Intervention Strategies: Fourth Edition. Belmont, CA: Wadsworth/Thomson Learning. 2001.

7. Substance Abuse and Mental Health Services Administration Crisis services: Effectiveness, cost-effectiveness, and funding strategies (HHS Publication No. (SMA)-14-4848). Rockville, MD: Author. 2014.

8. Thinn DS, Kuswanto CN, Sum MY, et al. The 23-hour observation unit admissions within the emergency service at a national tertiary psychiatric hospital: Clarifying clinical profiles, outcomes, and predictors of subsequent hospitalization. The Primary Care Companion for CNS Disorders. 2015; 17. Doi: 10.4088/PCC.15m01789

9. Gilli,PM,HilardJR,BellJ,etal. The psychiatricemergencyservice holding area: Effect on utilization of inpatient resources. American Journal of Psychiatry. 1989; 146: 369-372.

10. Francis E, Marchand W, Hart M, et al. Utilization and outcome in an overnight psychiatric observation program at a Veterans Affairs Medical Center. Psychiatric Services. 2000; 51(1): 92-95.

11. Heyland M, Emery C, Shattell M. The living room, a community crisis respite program: Offering people in crisis an alternative to emergency departments. Global Journal of Community psychology Practice. 2013; 4(3): 1-8.

12. National Action Alliance for Suicide Prevention: Clinical Care and Intervention Task Force. Suicide care in systems framework. Washington, DC: Education Development Center, Inc. Retrieved. 2011. from http://actionallianceforsuicideprevention. org/sites/actionallianceforsuicideprevention.org/files/taskforce/ ClinicalCareInterventionReport.pdf

13. Colombo A, Bendelow G, Fulford B, et al. Evaluating the influence of implicit models of mental disorder on processes of shared decision making within community-based multi-disciplinary teams. Social Science \& Medicine. 2003; 56(7): 1557-1570.

14. Collins C, Hewson DL, Munger R, et al. Evolving models of behavioral health integration in primary care. Milbank Memorial Fund. New York, NY. Milbank Memorial Fund. 2010. Retrieved from: https:// www.milbank.org/publications/evolving-models-of-behavioralhealth-integration-in-primary-care/

15. Heyland M, Johnson M. Evaluating an alternative to the emergency department for adults in mental health crisis. Issues in Mental Health Nursing. 2017; 38(7): 557-561.

16. Centers. Open Minds Management Newsletter. Retrieved from: https://www.openminds.com/market-intelligence/editorials/ psychiatric-boarding-evolving-models-community-crisis-centers. $\mathrm{htm} /$ 